\title{
Motion corrected Cadence CPS ultrasound for quantifying response to vasoactive drugs in a rat kidney model
}

\author{
Rachel E. Pollard ${ }^{\mathrm{a}}$, Paul A. Dayton ${ }^{\mathrm{b}, \mathrm{d}}$, Katherine D. Watson ${ }^{\mathrm{b}}$, Xiaowen Hu ${ }^{\mathrm{b}}$, Ismayil M. \\ Guracarc, and Katherine W. Ferrarab \\ a Department of Surgical and Radiological Sciences, School of Veterinary Medicine, University of \\ California, Davis \\ bDepartment of Biomedical Engineering, University of California, Davis \\ 'Siemens Medical Solutions, Ultrasound Division, Mountain View, CA
}

\begin{abstract}
Objective: To establish the ability of contrast enhanced motion corrected Cadence Pulse Sequencing (CPS) to detect changes in renal blood flow induced by vasoactive substances in rats.

Methods: Ultrasound contrast media was administered as a constant rate infusion into a phantom at a known rate and CPS data acquired. Rats were anesthetized and pre-drug CPS estimates of replenishment rate were made for the right kidney. Real-time motion correction was applied and parametric images were generated from the CPS data. Group 1 rats $(n=7)$ were administered a vasodilator and Group 2 rats $(n=3)$ were given a vasoconstrictor. CPS imaging of the kidney was repeated following ample time for drug effects to occur.
\end{abstract}

Results: Contrast CPS accurately estimated flow velocity in the phantom model. In addition, CPS defined statistically significant differences between pre and post drug blood flow in the renal medulla (vasodilator, $\mathrm{p}<0.01$; vasoconstrictor, $\mathrm{p}<0.0001$ ) and cortex (vasoconstrictor, $\mathrm{p}<0.0001$ ).

Conclusions: We conclude that motion corrected CPS ultrasound provides real time quantification of renal blood flow alterations and may prove useful for the assessment of blood flow in transplanted kidneys.

\section{Keywords}

CPS; ultrasound contrast; motion correction; renal blood flow

\section{Introduction}

Non-invasive imaging methods such as MR angiography ${ }^{1}, \mathrm{CT}$ angiography ${ }^{2}$ and Doppler ultrasound ${ }^{3}$ are used to assess renal vascular health in order to guide clinical judgements regarding management of people with kidney disease. Color and spectral Doppler sonography

(C) 2009 Elsevier Inc. All rights reserved.

Corresponding author: Rachel E. Pollard, DVM, PhD Department of Surgical and Radiological Sciences University of California, Davis School of Veterinary Medicine Davis, CA 95616 USA (530) 752-2511 (530) 752-6042 fax repollard@ ucdavis.edu. d Department of Biomedical Engineering, University of North Carolina, Chapel Hill (current address)

Publisher's Disclaimer: This is a PDF file of an unedited manuscript that has been accepted for publication. As a service to our customers we are providing this early version of the manuscript. The manuscript will undergo copyediting, typesetting, and review of the resulting proof before it is published in its final citable form. Please note that during the production process errors may be discovered which could affect the content, and all legal disclaimers that apply to the journal pertain. 
are frequently used methods by which to assess renal perfusion non-invasively and have proven particularly useful for the assessment of functional and compromised renal transplants 4,5 . Transplant perfusion is assessed using measures of resistive and perfusion index. However, these measures are only useful for the assessment of large arteries and are insensitive to alterations in vascular perfusion at the microvascular level ${ }^{6}$. With the addition of microbubble contrast agents, ultrasound becomes sensitive to capillary-sized vessels and very low flow rates while maintaining the ability to detect morphological information from traditional B-mode imaging. Ultrasound contrast agents are composed of high molecular weight gases encapsulated in an albumin, polymer or lipid shell ${ }^{7}$. These agents are strictly intravascular thus eliminating the need to differentiate intra from extravascular contrast agent. A variety of contrast specific pulse sequences designed to enhance non-linear echoes that are specifically produced by microbubble contrast agents have been developed and employed for the interrogation of regions of myocardial infarction, tumor microvasculature, and responses to therapy in both clinical and research settings $6,8-11$.

Another feature of ultrasound contrast agents is that they are destroyed when subjected to ultrasound of appropriate frequency and sufficient pressure ${ }^{7}$. A destructive pulse of ultrasound can be used to fragment the contrast agent within the region being imaged. Lower pressure, higher frequency, non-destructive pulses can be used to visualize the replenishment of intravascular contrast agent into the imaging region. The echo amplitude can be measured as the contrast agent re-fills the region and the rate of return can be estimated. Such a destructionreplenishment method was described as early as 1998, where Wei et al proposed the method for the assessment of blood flow velocity in the myocardium ${ }^{11}$. Continued research has been performed in the assessment of replenishment after acute myocardial infarction ${ }^{11}$ and in abdominal organs such as kidney and liver 6,12 .

Cadence Contrast pulse sequencing (CPS) is a non-linear ultrasound contrast imaging technique which uses a multipulse transmit sequence with precise changes in interpulse amplitude and phase that when received and combined allow for the rejection of linear (tissue) echoes while non-linear contrast echoes are retained ${ }^{13}$. The effect is of a real-time positive contrast angiogram. This technique has been used for the qualitative assessment of regional brain and solid organ infarction, liver and renal masses, and response to radiofrequency ablation of tumors in people ${ }^{14-18}$.

As with all ultrasound contrast imaging sequences, the use of CPS for the quantification of vascular parameters is compromised by tissue motion. Speckle tracking algorithms are methods for directly tracking the backscattered echoes produced by ultrasonic scatterers in blood or tissue ${ }^{19}$. Speckle patterns remain relatively constant as the tissue moves so that they can be tracked by pattern matching techniques. Several different speckle tracking techniques have been described however many come at a high computational cost directly related to the size of the search region and tracking kernel ${ }^{20-22}$. For real-time imaging, the sum absolute difference (SAD) method has been suggested because it is computationally simpler than other algorithms while still performing well in quantitative phantom experiments of motion tracking ${ }^{23}$.

The purpose of this study was to test the ability of motion corrected CPS combined with a destruction-replenishment scheme to quantify responses of the renal vasculature to vasoactive substances in normal rats. This native kidney model was chosen because this is a proof of concept study and, should changes in blood flow not be quantifiable in normal kidneys, the system would be unlikely to detect changes in diseased or transplanted kidneys were flow alterations may be subtle. SAD motion correction with destruction-replenishment CPS is a novel combination of features never before described for the real-time quantification of renal blood flow velocity. We hypothesized that this technique would prove to be sensitive and repeatable for the quantification of renal blood flow alteration. The goal of this pilot project is 
to determine if this new system can provide real-time quantification of changes in renal blood flow with outstanding repeatability so as to ultimately allow for the detection of cortical hypoperfusion in compromised kidneys. We anticipate this system will be useful initially as a research tool in rodents intended to further the understanding of renal blood flow in native and transplanted kidneys with potential application in clinical patients in the future.

\section{Materials and Methods}

\section{In Vitro Phantom Study}

30 silicone tubes (Dow Corning, Midland, MI) were bound together to form a bundle so as to mimic a vascular network. Both ends of the bundle were sealed into two small cylinder-shaped chambers, which hold $0.35 \mathrm{~mL}$ of liquid. Each tube had an inner diameter of $0.635 \mathrm{~mm}$ and outer diameter of $1.1938 \mathrm{~mm}$. Assuming a volume within the phantom tubes of $0.6 \mathrm{~mL}$ with a cross-sectional area of $6 \mathrm{~mm}^{2}$ and a syringe pump flow rate of $24 * 10^{-3} \mathrm{~mL} / \mathrm{s}$, we estimated a linear velocity of $\sim 4 \mathrm{~mm} / \mathrm{s}$ in the phantom tubes. Acoustic coupling gel (Aquasonic, Parker Labs, Fairfield, N.J.) was used to couple the transducer to the phantom. The phantom was placed on top of an ultrasound gel pad (Aquasonic, Parker Labs, Fairfield, N.J.). Contrast agents were produced in our laboratory as described previously 24 ; the agents were designed to have similar properties and a similar formulation to lipid-shelled agents used in the in vivo studies. The contrast agent was continuously injected at a speed of $4 \mathrm{~mm} / \mathrm{sec}$, which ranged within the biological flow rate set by a syringe pump (Harvard Apparatus, Holliston, MA). The ultrasound machine parameters were set to optimize the CPS image quality ( $7 \mathrm{MHz}$; CPS gain $=-20 ; 0.28$ MI) during application of the destruction-replenishment technique. The position of the transducer and the phantom is shown in Figure 1, with an azimuthal window of $14 \mathrm{~mm}$.

\section{Animals}

Ten adult, male outbred, albino Sprague-Dawley ${ }^{\circledR}$ rats (Harlan Sprague Dawley Inc., Indianapolis, IN) were imaged during these studies. Rats were approximately 450-500g each. Animal use protocols were approved by the Institutional Animal Care and Use Committee of the University of California, Davis. All animals were housed in a standard animal facility, fed a standard diet and given free access to food and water.

\section{In Vivo Ultrasound Imaging Protocol}

Ultrasound was performed using an Acuson Sequoia $512^{\circledR}$ system (Siemens Medical Solutions USA, Inc., Ultrasound Division, Issaquah, WA) with a 15L8 linear array transducer. Animals were anesthetized initially by placing each rat into an induction chamber and introducing 5\% aerosolized isoflurane in oxygen. Once anesthetized, the rat was removed from the chamber and anesthesia was maintained by titrating isoflurane $/ \mathrm{O}_{2}$ using a nose mask. Prior to imaging, the abdomen of each rat was shaved and further depiliated using Nair (Church \& Dwight, Co. Princeton, N.J.). Acoustic coupling gel (Aquasonic, Parker Labs, Fairfield, N.J.) was used to couple the transducer to the skin surface. A sagittal image of the right kidney was obtained and the transducer was mechanically fixed in position with an articulated arm. Definity (BristolMyers Squibb, N. Billerica, MA) was activated by shaking according to the manufacturer's instructions and diluted with $100 \mu \mathrm{L}$ to $600 \mu \mathrm{L}$ sterile saline in a $3 \mathrm{cc}$ syringe immediately before injection. Contrast agent was injected at 60 microliters/minute using a syringe pump (Harvard Apparatus, Holliston, MA) through a 24 gauge tail vein catheter with injection port. The continuous-rate injection was initiated 2 minutes prior to each imaging sequence to allow circulating contrast concentration to stabilize. Parametric maps of replenishment were acquired in real-time using Siemens Cadence ${ }^{\mathrm{TM}}$ contrast pulse sequencing (CPS) motion sensing probe (MSP) pulse sequencing and processing, which involved transmission of a low frequency, high-MI destructive pulse $(7 \mathrm{MHz}, 1.9 \mathrm{MI})$ which cleared the sample volume of contrast agent, followed by a series of lower MI ( $7 \mathrm{MHz}, 0.28 \mathrm{MI})$ pulses to image contrast as the kidney was 
reperfused. Automated MSP processing involved frame alignment using a real-time sum of absolute difference based search based on the derived B-mode information, and then further mapping of the colors of each pixel according to time to reach an intensity threshold set to $20 \%$ of full scale. The colormap was a non-linear map designed to provide high sensitivity to small differences in fast blood flow (fast arrival time), and lower sensitivity to changes in slow blood flow (slow arrival time). The arrival time was determined on a pixel by pixel basis by assigning each pixel a time value based on the elapsed time from the destruction event to the time that the CPS signal reaches $20 \%$ of the maximum value. The arrival time for pixels that had achieved the required signal level was then color-coded. Final images were recorded and saved for further offline processing to quantify replenishment in designated regions. The gain setting (CPS gain=10) and depth $(4 \mathrm{~cm})$ were left constant in all studies.

\section{Drug administration}

Imaging data was acquired prior to vasoactive drug administration. Changes in renal blood flow were subsequently mediated by administration of either the vasodilator hydralazine $(\mathrm{n}=7$ rats) or the vasoconstrictor dopamine (Dopamine, Abbott Laboratories, Chicago, IL) ( $\mathrm{n}=3$ rats). Hydralazine $(5 \mathrm{mg} / \mathrm{kg})$ was administered as a bolus. Imaging was performed 30 minutes after Hydralazine administration to allow for the drug to take effect. Only a single value was recorded for each animal pre and post drug. Dopamine was administered at a constant rate infusion (10 $\mu \mathrm{g} / \mathrm{kg} / \mathrm{min}$ ) via a second tail vein catheter over 12 minutes. Imaging was repeated during Dopamine administration but after a 10-minute delay to allow for the drug to take effect. At least 4 image acquisitions were performed prior to and after Dopamine administration. This was specifically done so as to assess parameter repeatability. Once imaging procedures were completed, rats were recovered from anesthesia and returned to the vivarium.

\section{Post-Processing}

Captured images were post-processed in MATLAB (Mathworks, Natick, MA). Regions of interest (ROIs) were manually selected around the entire cortex, the cortex and medulla of the kidney. Mean time to 20\% (TT20\%) maximum enhancement for the entire kidney, the medulla, and the cortex were derived from the time value of each pixel within the ROIs based on the colormap. The percent change in TT20\% was calculated between the pre and post drug values for the entire kidney, the cortex and the medulla.

\section{Statistical Analysis}

Statistical analysis was performed using commercially available software (Excel, Microsoft Corporation, Redmond, WA). Mean ( \pm standard deviation) values were calculated for each animal in the Dopamine group so as to assess the repeatability of parameter estimation within a given individual. Then the mean ( \pm standard deviation) values from all animals in each group were pooled and a Student t-test was used to evaluate for significant differences between the pre and post drug TT20\% values for the entire kidney, the cortex and the medulla. A p-value $<0.05$ was considered significant.

\section{Results \\ Phantom Study}

Based on the color map we designed for the destruction-replenishment technique, the light yellow color indicated fast arriving flow; red and pink colors indicated slow arriving flow (Figure 2). The distribution of the color in the perfused region showed the flow direction and agrees with the flow direction and flow rate set in the experiment. The arrival times in each ROI are plotted in Figure 2, which vary across the image from less than 1 second to 3.5 seconds. 
This corresponds with an estimated linear velocity of $4 \mathrm{~mm} / \mathrm{s}$ which equals the true velocity set for the syringe pump $\left(\mathrm{R}^{2}=0.91\right)$.

\section{Drug administration studies}

The mean $( \pm$ SD) TT20\% for the entire kidney prior to vasoactive drug administration in the Dopamine group $(\mathrm{n}=3)$ was $0.72( \pm 0.04) \mathrm{s}$. The mean TT20\% for the renal cortex and medulla prior to vasoactive drug administration in the Dopamine group was $0.47( \pm 0.02) \mathrm{s}$ and 1.06 $( \pm 0.07) \mathrm{s}$, respectively. The mean TT20\% for the entire kidney during Dopamine administration $(\mathrm{n}=3)$ was $0.63( \pm 0.05) \mathrm{s}$, which represented a $12.5 \%$ decrease in replenishment time and was statistically significantly different from the pre-Dopamine values $(\mathrm{p}<<<0.0001)$. The mean TT20\% for the renal cortex and medulla during Dopamine administration was 0.43 $( \pm 0.01) \mathrm{s}(8.5 \%$ decrease $)$ and $0.88( \pm 0.12) \mathrm{s}(17 \%$ decrease $)$, respectively. Both parameters were significantly different from the pre-Dopamine values ( $\mathrm{p}<<<0.0001$ for both). Values for individual rats are shown in Figure 3 (a-c). Note the small standard error bars representing the variability in the 4 different measurements acquired in each individual rat implying good repeatability. Parametric images show differences in renal replenishment times during Dopamine administration (Figure $3 \mathrm{~d}, \mathrm{e}$ ).

The mean $( \pm$ SD) TT20\% for the entire kidney prior to vasoactive drug administration in the Hydralazine group $(n=7)$ was $0.64( \pm 0.17)$ s. The mean TT20\% for the renal cortex and medulla prior to vasoactive drug administration in the Hydralazine group was $0.46( \pm 0.03) \mathrm{s}$ and 0.95 $( \pm 0.28)$ s, respectively. The mean TT20\% for the entire kidney after Hydralazine administration $(\mathrm{n}=7)$ was $0.83( \pm 0.24) \mathrm{s}$, which represented a $29.7 \%$ increase in replenishment time and was statistically significantly different from the pre-Hydralazine values $(\mathrm{p}=0.02)$. The mean TT20\% for the renal cortex and medulla during Hydralazine administration was $0.54( \pm$ $0.10) \mathrm{s}(17.4 \%$ increase) and $1.32( \pm 0.45) \mathrm{s}(38.9 \%$ increase), respectively. Significant differences were found in comparison to the pre-Hydralazine medullary values $(\mathrm{p}=0.01)$ but not the cortical values $(\mathrm{p}=0.10)$. Values for individual rats are shown in Figure $4(\mathrm{a}-\mathrm{c})$. Parametric images show differences in renal replenishment times during Hydralazine administration (Figure $4 \mathrm{~d}, \mathrm{e}$ ).

\section{Comments}

The results of this study indicate that motion corrected CPS ultrasound combined with a destruction-replenishment scheme is capable of estimating true velocity in a phantom model. When estimating replenishment time, this system accurately depicted fast replenishment in the region closest to the syringe pump input and depicted slower replenishment in the further regions. Moreover, good correlation was found between true velocity and parametric estimates in vitro. This system also appears capable of quantifying significant changes in renal cortical and medullary blood flow velocities in vivo induced by the systemic administration of vasoconstrictive or vasodilatory substances. The kidney is highly regulated so that systemic changes in vascular tone and blood pressure will result in modifications of renal blood flow with the end goal being maintenance of glomerular filtration ${ }^{25}$. Thus, if the systemic and renal vasculature is constricted, flow velocity (replenishment) must increase so as to maintain overall blood flow. Likewise, if the systemic and renal vasculature is dilated, flow velocity will decrease. Dopamine is known to increase renal blood flow related to increased flow velocity in constricted vessels. Hydralazine is known to decrease renal blood flow related to a decrease in flow velocity in dilated vessels.

Our results show that this system was capable of identifying such changes. In fact, a similar study using contrast enhanced MRI to evaluate the effect of vasomodulatory drugs on blood flow in a variety of tissues in rats was less sensitive to alterations in renal blood flow velocity with the administration of hydralazine although overall renal blood flow decreased by $26 \%$ 
26. The administration of a different vasoconstrictor (histamine) resulted in a $68 \%$ increase in renal blood flow velocity in that study which may be a reflection of the difference in the 2 drugs.

Previous authors have used contrast ultrasound techniques to evaluate blood flow in normal and diseased native kidneys ${ }^{27}$ but predominantly in functional and compromised transplanted kidneys ${ }^{28-32}$. We believe this ultrasound imaging system is novel in several ways potentially providing more reliable data. First, the SAD motion correction algorithm reduces the effect of tissue and respiratory motion upon parameter generation. Motion correction allows frames over a long period of time to be combined using a maximum intensity projection or to compute spatially varying contrast agent arrival time. Tissue motion is detrimental to image quality and severely limits the accuracy of ROI calculations. Particularly in the cranial abdomen, respiratory motion can be quite dramatic such that an ROI may contain cortical tissue at inspiration and medullary tissue at expiration. Given the differences between cortical and medullary flow, this would dramatically influence TT20\% or other blood flow measurements. It is important to note that this motion correction algorithm accounts for in-plane motion but not for out-of-plane motion. Based on the typical pattern of rodent respiration, in-plane motion in the cranial and caudal directions predominates. However, the effect of out-of-plane motion on parameter estimation is not known and must be addressed in future studies.

Second is the introduction of an automated destruction-replenishment scheme in combination with CPS. Parametric images were generated during the imaging session, which allowed clear differentiation between cortical and medullary tissue within the kidney. This streamlined the qualitative assessment of the imaging data and minimized the post-processing time. A nonlinear colormap was chosen for data acquisition in the kidney because we expected rapid replenishment to dominate. By distributing the colormap in a way that amplified small differences between rapidly replenished areas, this system was capable of detecting more subtle flow differences and alterations.

We chose to evaluate the TT20\% parameter as a robust parameter that can be independent of attenuation. Since this is a relative measure of the change in the echo intensity over the transmitted imaging pulse train, it may overcome some limitations of previous efforts to quantify microvascular flow with ultrasound. More specifically, this parameter is not likely to be affected by slight differences in bubble concentration nor does it rely on the questionable assumption that signal intensity is directly related to bubble (and therefore blood) volume.

Indeed, as shown by the low standard deviation between repeated measurements within a given rat, it appears that this parameter is highly reproducible if the transducer is not moved.

It is important to note that it was not the intent of this study to establish a range of values representative of normal renal blood flow. In fact, when looking at the pre-drug data, there was substantial inter-individual variation of the TT20\% values. This may indicate true interindividual variation or represent noise within the system when applied in vivo. Although little noise was detected during the phantom study, this question could only be answered by comparison of the TT20\% value to an in vivo gold standard. While it is possible that a range of normal TT20\% replenishment values can be established, the change in this parameter over time within a given individual may ultimately be more useful and interesting.

A limitation and concern not addressed in this study involves the relative difficulty of exactly reproducing the same imaging plane during serial studies so that variations within the kidney itself are accounted for. As such, one must be certain that an adequate representation of regional tissue blood flow is sampled in order to eliminate the possibility that the choice of imaging plane is influencing the outcome during sequential examinations. One possible way to overcome this limitation is by averaging quantitative values over several imaging planes. This 
may provide a more accurate regional estimate of blood flow and eliminate the need to exactly reproduce the imaging plane used in previous examinations. Additional studies will be necessary to determine if this will sufficiently address the limitation of imaging plane reproducibility. Alternatively, 2D arrays are currently in various stages of development and application. The combination of real time 3D imaging with destruction-replenishment CPS would allow for the global assessment of tissue or organ blood flow from which quantitative data could be extracted from, for example, the entire renal medulla. It is likely that this will ultimately be necessary prior to advancement into clinical application in order to reduce the error introduced by the inability to reproduce the same imaging plane.

Another limitation of this study is that blood pressure changes were not recorded in these animals. Non-invasive, accurate blood pressure measurement is notoriously difficult in rats. Blood pressure and heart rate monitoring may be useful during future studies using this technique to ensure that the drugs are taking the expected effect and as a gauge for the anticipated effect on renal blood flow.

\section{Conclusions}

Motion corrected destruction-replenishment CPS ultrasound appears to be a non-invasive method for quantifying renal blood flow velocity. Quantitative parameters derived from motion corrected ROI analysis appear to be robust when the imaging plane is not altered while the acquisition of multiple imaging planes or the implementation of a $2 \mathrm{D}$ array transducer may be necessary to apply this technique in serial tissue assessment. Although still developmental, this technique shows promise as a pre-clinical method for monitoring changes in renal blood flow velocity over time. Additional studies will be necessary to further define the role of motion corrected CPS destruction-replenishment ultrasound for the assessment of renal disease and in complicated renal transplants.

\section{Acknowledgements}

This study was funded by NCI grant number CA76062.

\section{References}

1. Jain R, Sawhney S. Contrast-enhanced MR angiography (CE-MRA) in the evaluation of vascular complications of renal transplantation. Clin Radiol 2005;60(11):1171-81. [PubMed: 16223613]

2. Tunaci A, Yekeler E. Multidetector row CT of the kidneys. Eur J Radiol 2004;52(1):56-66. [PubMed: 15380847]

3. Friedewald SM, Molmenti EP, Friedewald JJ, Dejong MR, Hamper UM. Vascular and nonvascular complications of renal transplants: sonographic evaluation and correlation with other imaging modalities, surgery, and pathology. J Clin Ultrasound 2005;33(3):127-39. [PubMed: 15756666]

4. Radermacher J, Mengel M, Ellis S, et al. The renal arterial resistance index and renal allograft survival. N Engl J Med 2003;349(2):115-24. [PubMed: 12853584]

5. Sommerer C, Hergesell O, Nahm AM, et al. Cyclosporin A toxicity of the renal allograft--a late complication and potentially reversible. Nephron 2002;92(2):339-45. [PubMed: 12218312]

6. Wei K, Le E, Bin JP, Coggins M, Thorpe J, Kaul S. Quantification of renal blood flow with contrastenhanced ultrasound. J Am Coll Cardiol 2001;37(4):1135-40. [PubMed: 11263620]

7. Chomas JE, Dayton P, Allen J, Morgan K, Ferrara KW. Mechanisms of contrast agent destruction. IEEE Trans Ultrason Ferroelectr Freq Control 2001;48(1):232-48. [PubMed: 11367791]

8. Bartolotta TV, Taibbi A, Midiri M, De Maria M. Hepatocellular cancer response to radiofrequency tumor ablation: contrast-enhanced ultrasound. Abdom Imaging. 2007

9. Pollard RE, Garcia TC, Stieger SM, Ferrara KW, Sadlowski AR, Wisner ER. Quantitative evaluation of perfusion and permeability of peripheral tumors using contrast-enhanced computed tomography. Invest Radiol 2004;39(6):340-9. [PubMed: 15167100] 
10. Sugimoto K, Moriyasu F, Kamiyama N, Yamada M, Iijima H. Correlation between parametric imaging using contrast ultrasound and the histological differentiation of hepatocellular carcinoma. Hepatol Res. 2007

11. Wei K, Jayaweera AR, Firoozan S, Linka A, Skyba DM, Kaul S. Quantification of myocardial blood flow with ultrasound-induced destruction of microbubbles administered as a constant venous infusion. Circulation 1998;97(5):473-83. [PubMed: 9490243]

12. Hancock J, Dittrich H, Jewitt DE, Monaghan MJ. Evaluation of myocardial, hepatic, and renal perfusion in a variety of clinical conditions using an intravenous ultrasound contrast agent (Optison) and second harmonic imaging. Heart 1999;81(6):636-41. [PubMed: 10336924]

13. Phillips P, Gardner E. Contrast-agent detection and quantification. Eur Radiol 2004;14(Suppl 8):P410. [PubMed: 15700327]

14. Bartels E, Henning S, Wellmer A, Giraldo-Velasquez M, Kermer P. Evaluation of cerebral perfusion deficit in stroke patients using new transcranial contrast imaging CPS technology--preliminary results. Ultraschall Med 2005;26(6):478-86. [PubMed: 16453219]

15. Bauditz J, Schade T, Wermke W. [Sonographic diagnosis of hilar cholangiocarcinomas by the use of contrast agents]. Ultraschall Med 2007;28(2):161-7. [PubMed: 17366374]

16. von Herbay A, Schick D, Horger M, Gregor M. [Low-MI-sonography with the contrast-agent SonoVue in the diagnosis of infarction of the spleen, kidney, liver and pancreas]. Ultraschall Med 2006;27(5):445-50. [PubMed: 17033946]

17. Wink MH, de la Rosette JJ, Laguna P, Lagerveld BW, Wijkstra H. Ultrasonography of renal masses using contrast pulse sequence imaging: a pilot study. J Endourol 2007;21(5):466-72. [PubMed: 17523897]

18. Wink MH, Laguna MP, Lagerveld BW, de la Rosette JJ, Wijkstra H. Contrast-enhanced ultrasonography in the follow-up of cryoablation of renal tumours: a feasibility study. BJU Int 2007;99(6):1371-5. [PubMed: 17355368]

19. Bohs LN, Geiman BJ, Anderson ME, Gebhart SC, Trahey GE. Speckle tracking for multidimensional flow estimation. Ultrasonics 2000;38(18):369-75. [PubMed: 10829690]

20. Jiang J, Hall TJ. A parallelizable real-time motion tracking algorithm with applications to ultrasonic strain imaging. Phys Med Biol 2007;52(13):3773-90. [PubMed: 17664576]

21. Li PC, Lee WN. An efficient speckle tracking algorithm for ultrasonic imaging. Ultrason Imaging 2002;24(4):215-28. [PubMed: 12665238]

22. Zhu Y, Hall TJ. A modified block matching method for real-time freehand strain imaging. Ultrason Imaging 2002;24(3):161-76. [PubMed: 12503771]

23. Bohs LN, Trahey GE. A novel method for angle independent ultrasonic imaging of blood flow and tissue motion. IEEE Trans Biomed Eng 1991;38(3):280-6. [PubMed: 2066142]

24. Borden MA, Kruse DE, Caskey CF, Zhao S, Dayton PA, Ferrara KW. Influence of lipid shell physicochemical properties on ultrasound-induced microbubble destruction. IEEE Trans Ultrason Ferroelectr Freq Control 2005;52(11):1992-2002. [PubMed: 16422411]

25. Koeppen, B.; Stanton, B. Renal Physiology. Philadelphia: 2007.

26. Su MY, Wang Z, Roth GM, Lao X, Samoszuk MK, Nalcioglu O. Pharmacokinetic changes induced by vasomodulators in kidneys, livers, muscles, and implanted tumors in rats as measured by dynamic Gd-DTPA-enhanced MRI. Magn Reson Med 1996;36(6):868-77. [PubMed: 8946352]

27. Setola SV, Catalano O, Sandomenico F, Siani A. Contrast-enhanced sonography of the kidney. Abdom Imaging 2007;32(1):21-8. [PubMed: 17420958]

28. Cosgrove DO, Chan KE. Renal transplants: what ultrasound can and cannot do. Ultrasound Q 2008;24 (2):77, 87. [PubMed: 18528243] quiz 141-2

29. Fischer T, Dieckhofer J, Muhler M, et al. The use of contrast-enhanced US in renal transplant: first results and potential clinical benefit. Eur Radiol 2005;15(Suppl 5):E109-16. [PubMed: 18637238]

30. Fischer T, Filimonow S, Dieckhofer J, et al. Improved diagnosis of early kidney allograft dysfunction by ultrasound with echo enhancer--a new method for the diagnosis of renal perfusion. Nephrol Dial Transplant 2006;21(10):2921-9. [PubMed: 16822787]

31. Fischer T, Filimonow S, Rudolph J, et al. Arrival time parametric imaging: a new ultrasound technique for quantifying perfusion of kidney grafts. Ultraschall Med 2008;29(4):418-23. [PubMed: 17315111] 
32. Schwenger V, Zeier M. Contrast-enhanced sonography as early diagnostic tool of chronic allograft nephropathy. Nephrol Dial Transplant 2006;21(10):2694-6. [PubMed: 16877483] 

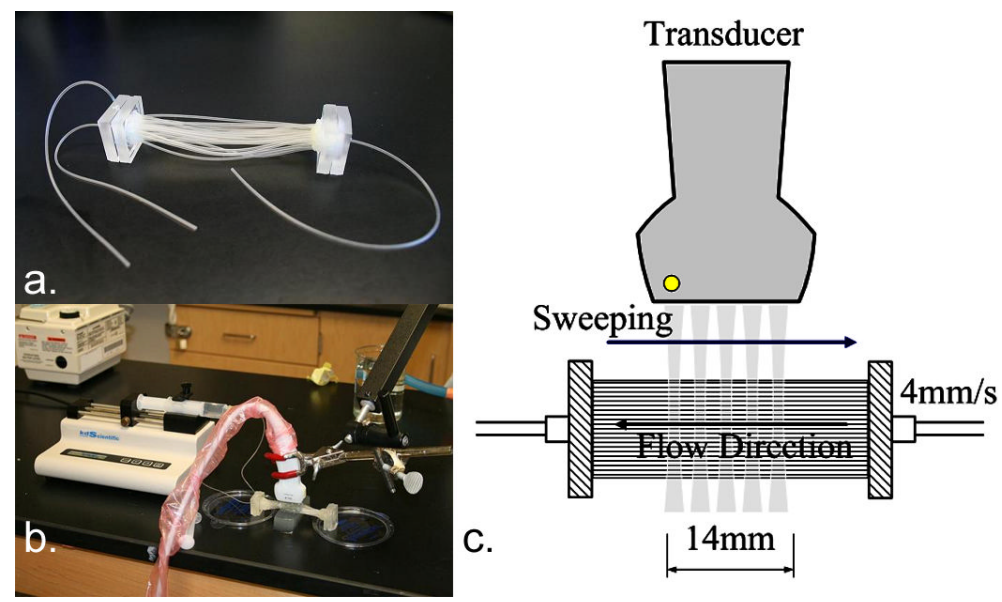

Figure 1.

a. The tube phantom is shown. b.The phantom is attached to a syringe pump for injection of contrast media at a controlled rate. The ultrasound transducer is coupled to the phantom using gel and fixed in place with a mechanical arm. c. A schematic is depicted showing the ultrasound transducer and the flow direction in the phantom tubes. 


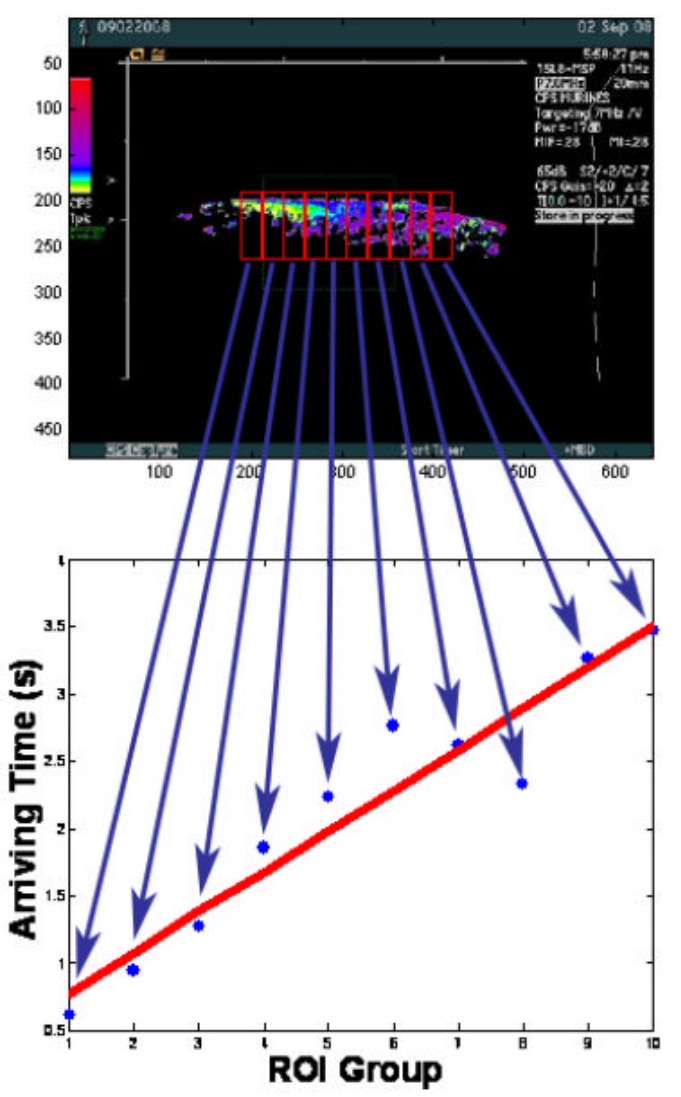

Figure 2.

The time to $20 \%$ replenishment is shown for the tube phantom imaged at a constant infusion rate of $4 \mathrm{~mm} / \mathrm{sec}$. The top parametric image shows the replenishment times and the regions of interest drawn over the tubes. When arrival time is plotted against the region of interest, it becomes clear that arrival times are longer for regions that are further from the input of the phantom. This shows that the system is measuring shorter and longer replenishment times accurately for a known flow velocity. 

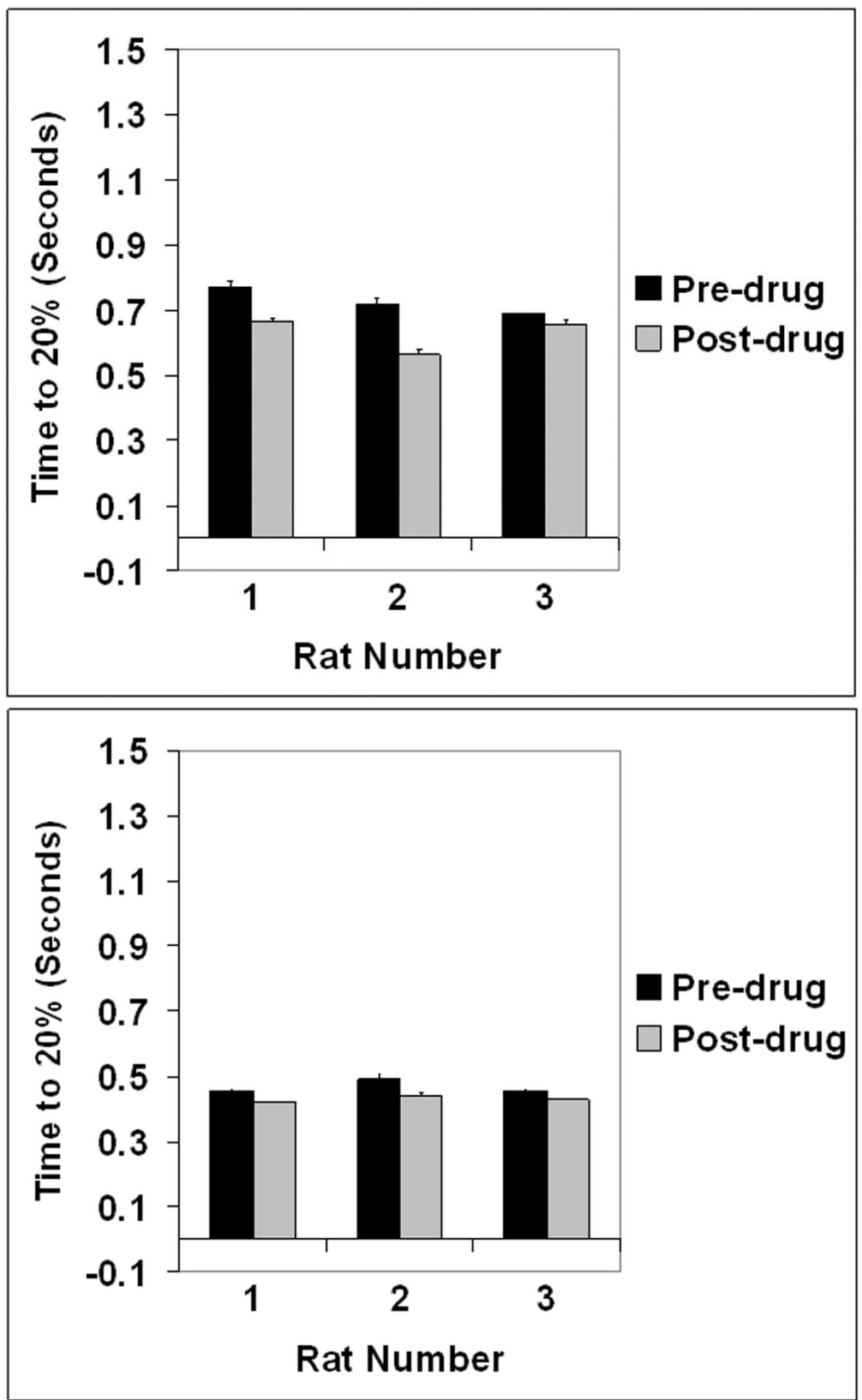


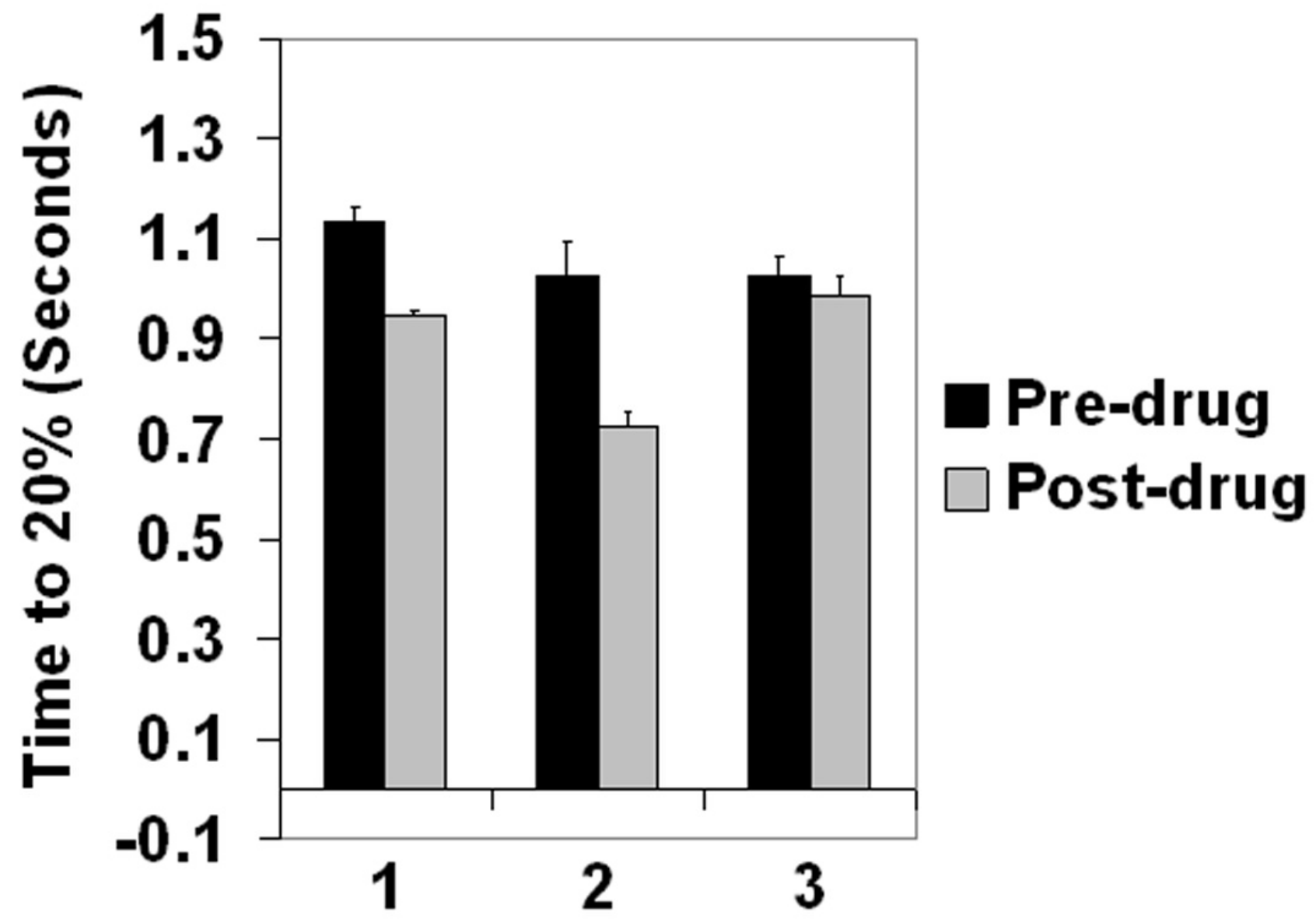

Rat Number
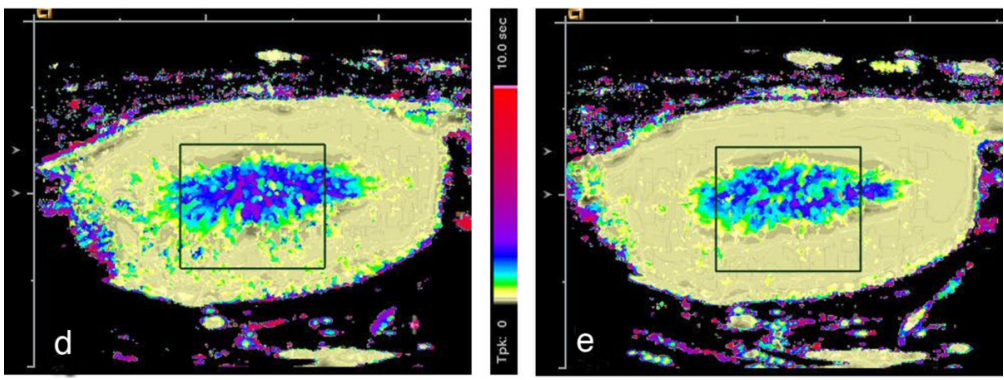

Figure 3.

a. The time to $20 \%$ maximum replenishment (TT 20\%) is shown for a region of interest (ROI) drawn around the entire kidney prior to and after administration of Dopamine. Note that all 3 rats showed a decrease in replenishment times indicating more rapid refill after Dopamine. Values shown represent a mean of 4 measurements taken at each time point in each rat with standard error bars demonstrating measurement repeatability. b. The TT $20 \%$ is shown for an ROI drawn in the renal cortex prior to and after administration of Dopamine. Note that all 3 rats showed a decrease in replenishment times indicating more rapid refill after Dopamine. 
Values shown represent a mean of 4 measurements taken at each time point in each rat with standard error bars demonstrating measurement repeatability. c. The TT $20 \%$ is shown for an ROI drawn in the renal medulla prior to and after administration of Dopamine. Note that all 3 rats showed a decrease in replenishment times indicating more rapid refill after Dopamine. Values shown represent a mean of 4 measurements taken at each time point in each rat with standard error bars demonstrating measurement repeatability. $\mathbf{d}$.A parametric image of the right kidney is shown prior to vasoactive drug administration. The colorbar shows time to $20 \%$ replenishment with the most rapid replenishment represented in yellow and the slowest replenishment represented in red. Notice that the colormap is non-linear with more color options present in the shortest replenishment time range. e. The same kidney is shown after administration of the vasoconstricting drug Dopamine. Cortical replenishment is now almost completely represented by yellow and medullary replenishment tends more toward green and blue (more rapid replenishment) in comparison to a. 


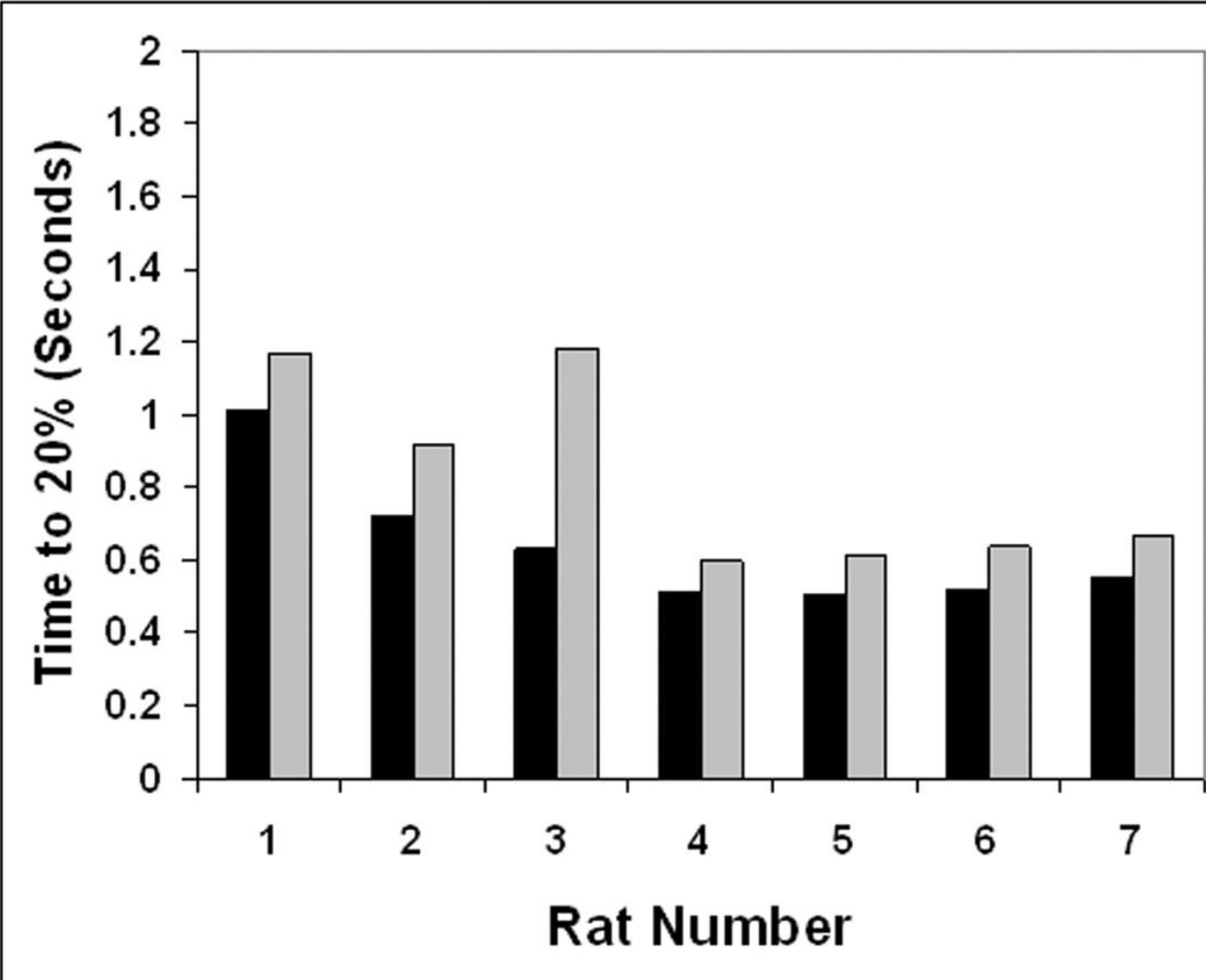

Pre-drug Post-drug

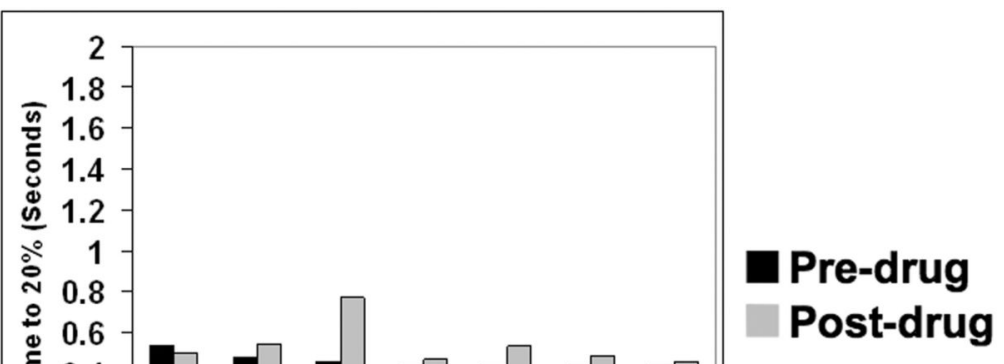




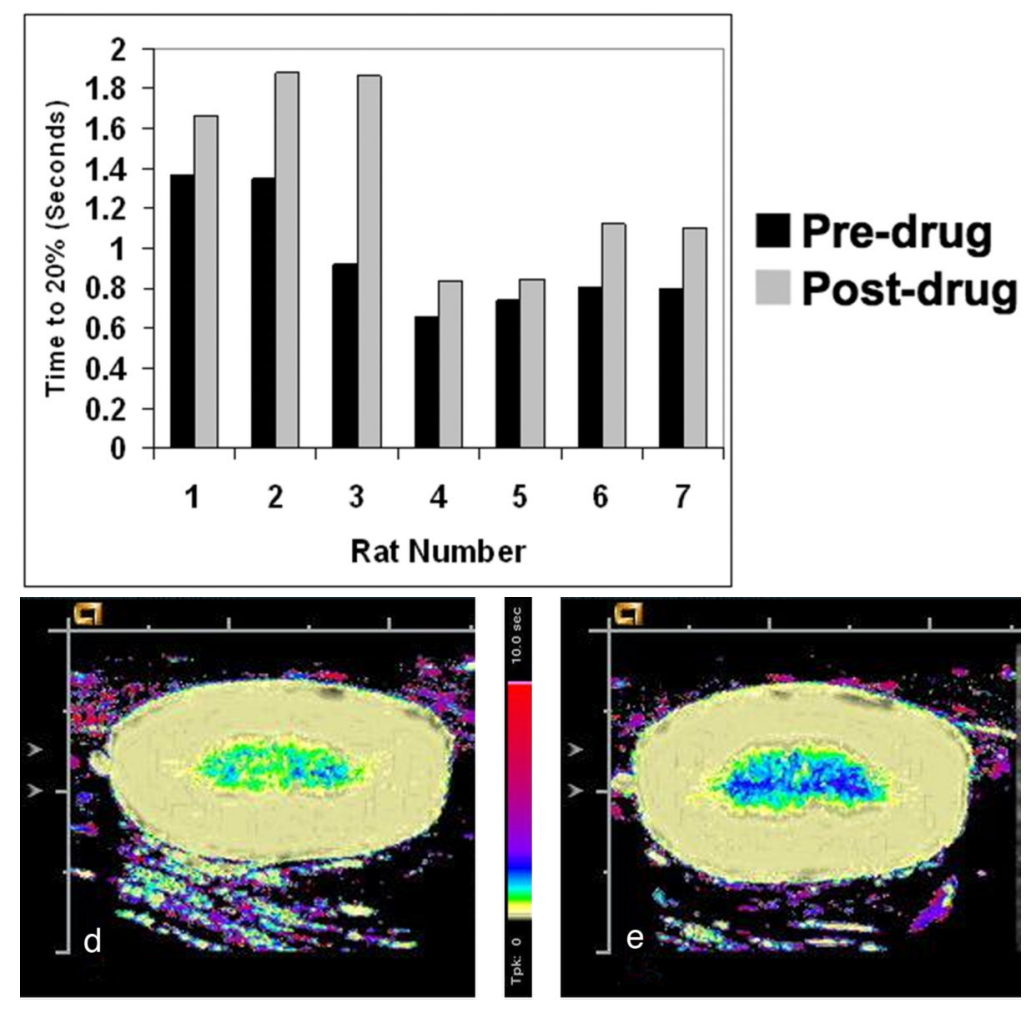

Figure 4.

a. The time to $20 \%$ maximum replenishment (TT 20\%) is shown for a region of interest (ROI) drawn around the entire kidney prior to and after administration of Hydralazine. Note that all 7 rats showed an increase in replenishment times indicating slower refill after Hydralazine.

b. The TT $20 \%$ is shown for an ROI drawn in the renal cortex prior to and after administration of Hydralazine. Note that all 7 rats showed an increase in replenishment times indicating slower refill after Hydralazine. c. The TT $20 \%$ is shown for an ROI drawn in the renal medulla prior to and after administration of Hydralazine. Note that all 7 rats showed an increase in replenishment times indicating slower refill after Hydralazine. d. A parametric image of the right kidney is shown prior to vasoactive drug administration. The colorbar shows time to $20 \%$ replenishment with the most rapid replenishment represented in yellow and the slowest replenishment represented in red. Notice that the colormap is non-linear with more color options present in the shortest replenishment time range. e. The same kidney is shown after administration of the vasodilatory drug Hydralazine. Cortical replenishment is relatively similar while medullary replenishment tends more dark blue and purple (slower replenishment) in comparison to a. 